\title{
Ring-based electron cooler for high energy beam cooling
}

\author{
H. Zhao ${ }^{*}{ }^{*}$ J. Kewisch, ${ }^{\dagger}$ M. Blaskiewicz, and A. Fedotov \\ Brookhaven National Laboratory, Upton, New York 11973, USA
}

(Received 28 December 2020; accepted 15 March 2021; published 5 April 2021)

\begin{abstract}
An electron-ion collider (EIC) at Brookhaven National Laboratory is being proposed as a new discovery machine for the nuclear physics and quantum chromodynamics. The hadron beam cooling plays an important role in the EIC machine to achieve its physics goals. The most challenging is cooling of protons at the highest energy in the EIC. In this paper, we present a possible design of a ring-based electron cooler for the high energy hadron beam cooling. In the proposed approach, the electrons will cool the hadrons while being cooled themselves by radiation damping in the storage ring. For the design of the cooler using the storage ring approach several aspects become very important, including electron ring optics design, chromaticity correction, calculating the dynamic aperture, radiation damping, quantum excitation, and intrabeam scattering. In addition, such effects as beam-beam scattering due to interaction of electrons with hadrons becomes of special concern, and we develop a generalized approach to it. In this paper, we take all of the above effects into the design, and discuss the beam lifetime and instabilities in the ring. A special feature of our design is an effective use of dispersion in the cooling section, both for the ions and electrons, to redistribute the cooling rate between the longitudinal and horizontal planes. Finally, the cooling performance is simulated for proton beam at the top energy of the EIC. Our conclusion is that such ringbased cooler could be a feasible approach to provide required parameters of hadron beam at the top energy of $275 \mathrm{GeV}$ for the EIC.
\end{abstract}

DOI: 10.1103/PhysRevAccelBeams.24.043501

\section{INTRODUCTION}

Electron cooling is a powerful method to shrink the size and momentum spread of the stored ion beams for accumulation and high-precision experiments. Since it was first proposed by Budker in 1967 [1], this technique has been widely applied and developed in many heavy ion accelerators around the world [2-5]. With the development of particle accelerators and the higher requirements for experimental physics, beam cooling with much higher energy electron beam is demanded. In 2005, the first relativistic electron cooling was demonstrated at Fermilab [6]. Recently, the world's first rf-based electron cooler was successfully commissioned at Brookhaven National Laboratory (BNL) and became the first electron cooler to directly cool colliding ion beams [7]. It provides the possibility to use a similar approach to develop high-energy electron coolers in the future.

Brookhaven National Laboratory (BNL) is designing an electron-ion collider (EIC), which will be a new discovery

\footnotetext{
*hezhao@bnl.gov

†jorg@bnl.gov
}

Published by the American Physical Society under the terms of the Creative Commons Attribution 4.0 International license. Further distribution of this work must maintain attribution to the author(s) and the published article's title, journal citation, and DOI. machine that opens new frontiers for the research in nuclear physics and quantum chromodynamics [8]. In order to maintain the high luminosity during long collision runs, it is desirable to cool the hadron beam to counteract the emittance growth caused by intrabeam scattering (IBS). There are several new cooling concepts proposed for the EIC, such as coherent electron cooling [9] and microbunched electron cooling [10]. In addition to these new concepts, conventional electron cooling schemes, such as the Energy Recovery Linac-based electron cooler [11] and ring-based electron cooler using dc electron beam [12], are also under consideration. In this paper, we present a design of the electron storage ring cooler with bunched electron beam for the EIC.

Electron cooling using a storage ring was considered before $[13,14]$. In our design we employ bunched electron beams without a continuous magnetic field in the cooling section, similar to [7], and make effective use of dispersive cooling by introducing dispersions for the ions and electrons in the cooling section.

The ring-based cooler approach is based on the conventional electron cooling technique, but the electrons which continuously interact with the hadron beam are being cooled by radiation damping in the storage ring. For a good cooling performance, the electron beam with low temperature and high intensity is needed, which requires a strong damping effect in the electron storage ring. Therefore, this concept strongly depends on electron ring 
design and is feasible only at high energies, at which the IBS heating is reduced while the radiation damping is enhanced. In the paper, we describe the ring optics design and achieved equilibrium parameters of electron beam due to the radiation damping, quantum excitation, IBS effect and beam-beam scattering (BBS). We also introduce a generalized treatment of the BBS and the 3D quantum lifetime, and make a simple estimation of the beam instabilities in the ring.

To calculate the cooling process, a cooling simulation code was developed, in which the 3D nonmagnetized cooling force, IBS and the dispersion effect on cooling are considered. It is also shown that the dispersions of hadron and electron beams can be effectively used to redistribute the horizontal and longitudinal cooling rates. Based on the simulation results, we conclude that such ring-based cooler with bunched electron beam could be a feasible approach to provide required parameters of proton beam at the top energy of $275 \mathrm{GeV}$ for the EIC.

The paper is organized as follows. In Sec. II, the main beam parameters in EIC and the cooling requirement are introduced. In Sec. III, the lattice design of the ring cooler is presented. In Sec. IV, the electron beam parameters in the ring are calculated and some important properties of the cooler are described. Then the cooling simulation results on hadron beam are presented in Sec. V. Finally, a summary is given in Sec. VI.

\section{COOLING REQUIREMENT}

In the design of beam cooling for the EIC, the most demanding case is to cool protons with the energy of $275 \mathrm{GeV}$. During the long collision stores, the emittance growth of proton beam due to IBS is the dominant limitation for the luminosity. The requirement for the hadron beam cooling is mainly to counteract the IBS heating effect. Table I summarizes the proton beam parameters which are relevant for the calculation of IBS growth rates and are used to specify the required cooling rates. The evolution of the $275 \mathrm{GeV}$ proton beam emittance

TABLE I. Proton beam parameters for cooling.

\begin{tabular}{lc}
\hline \hline Proton beam energy [GeV] & 275 \\
\hline Relativistic factor $\gamma$ & 293.1 \\
Number of protons per bunch & $6.88 \times 10^{10}$ \\
Number of bunches & 1160 \\
Average beam current [A] & 1 \\
rms emittance (x/y) [nm] & $9.6 / 1.5$ \\
rms momentum spread & $6.6 \times 10^{-4}$ \\
rms bunch length [cm] & 6 \\
Maximum space charge & 0.003 \\
Transverse IBS growth time (H/V) [hours] & $2.0 / 12$ \\
Longitudinal IBS growth time [hours] & 3.4 \\
Electron cooling beam energy [MeV] & 149.8 \\
Required cooling time [hours] & $\leq 2$ \\
\hline \hline
\end{tabular}

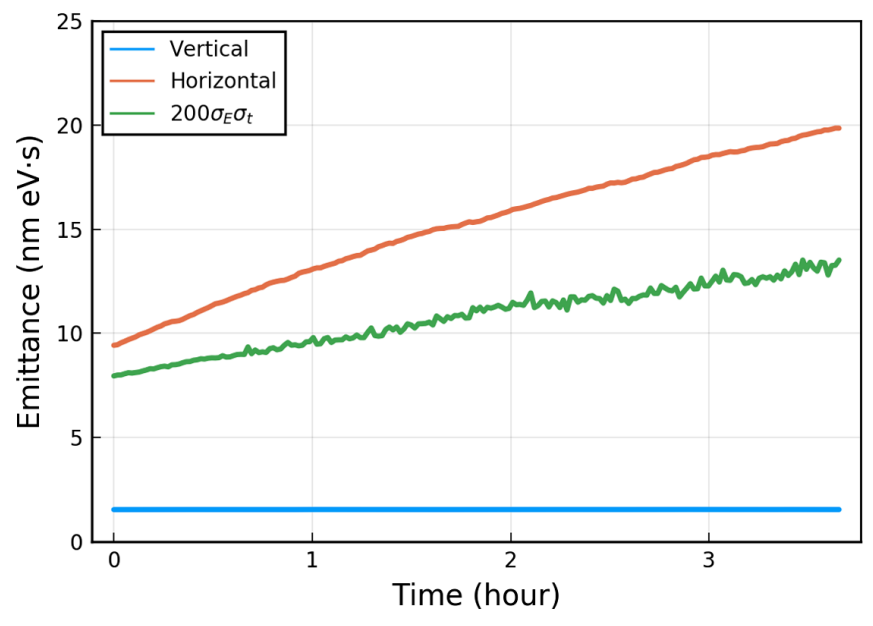

FIG. 1. Emittance growth of the $275 \mathrm{GeV}$ proton beam caused by IBS.

caused by IBS is shown in Fig. 1. It shows that the IBS heating effect for the flattened proton beam is dominated by the horizontal and longitudinal planes. As a result, vertical cooling is not needed for these parameters. We should note that required small vertical emittance of protons, shown in Table I, is assumed to be available at the start of the store, for example by precooling of protons at injection energy. Because vertical cooling is not required, one can effectively use horizontal dispersion to redistribute the cooling rates between the longitudinal and horizontal planes, and achieve required cooling performance.

\section{ELECTRON RING LATTICE OVERVIEW}

The electron storage ring has a race track shape, with the cooling section located in one long straight section and wigglers in the other. The ring is mirror symmetric around the center of the cooling section, and the top view of the ring is shown in Fig. 2. The cooling section has a length of $170 \mathrm{~m}$ and it fits into the straight section of the hadron ring.

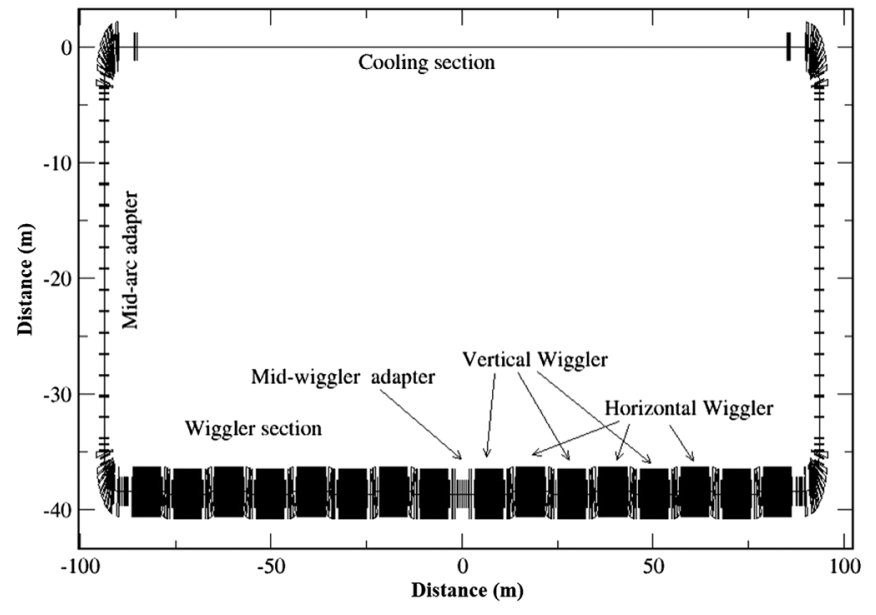

FIG. 2. Layout of the ring cooler. 


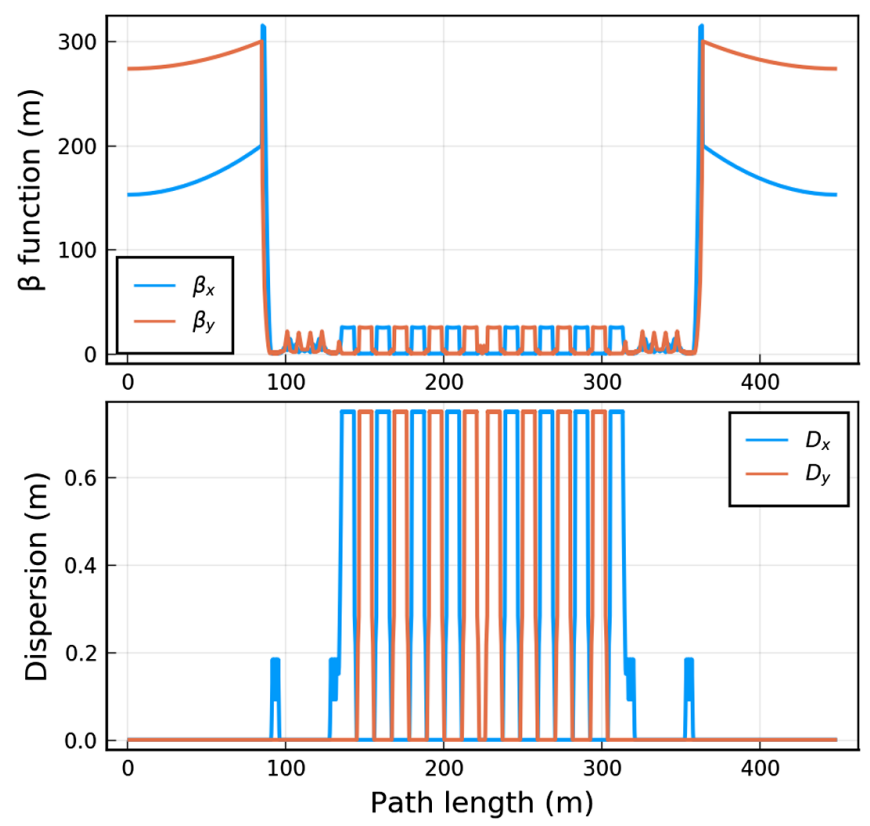

FIG. 3. Optics of the ring cooler.

There are four arcs with radius of $3.42 \mathrm{~m}$, each of them has ten dipoles and a 90 degree phase advance per cell. The mid-arc adapter is quadrupole section to adjust the tunes of the machine. The wiggler section is also mirror symmetric with four pairs of wigglers in each half. In our setup, the alternating horizontal and vertical wigglers are used, and the wiggler poles are shaped as combined function sector dipoles. The mid-wiggler adapter connects the two parts and is also used to optimize the high order chromaticity. The optics of the ring cooler is shown in Fig. 3 [15].

Because of the edge focusing of the dipoles, the wigglers will create a large chromaticity. The contribution of the arcs is relatively small and there are only a few slots available to place sextupoles. Simultaneously, the dispersion in the arc has to be kept small to avoid transverse emittance growth from IBS. This would require strong sextupoles decreasing the dynamic aperture significantly. In order to reduce the strength of the sextupoles, the sextupoles could be placed inside the wiggler magnets, where a small dispersion can be available throughout the length of the magnet, requiring smaller sextupole field strength. Also, since the edge focusing is only in one plane, a large ratio between horizontal and vertical beta functions can be maintained, making the chromaticity compensation more effective.

As shown in Fig. 4, there are two dipoles on each side of the wigglers to create dispersion. The dispersion is then amplified to the desired value by the following quadrupoles. Considering IBS, the increase of the transverse emittance, which is proportional to $H=\gamma D^{2}+2 \alpha D D^{\prime}+$ $\beta D^{\prime 2}$, must be minimized. The beta function in the wiggle plane is chosen to be $25 \mathrm{~m}$, which makes the $D^{\prime 2}$ term of the $H$ function 2 orders of magnitude bigger than the other terms. Therefore, the dispersion in the wiggler section does
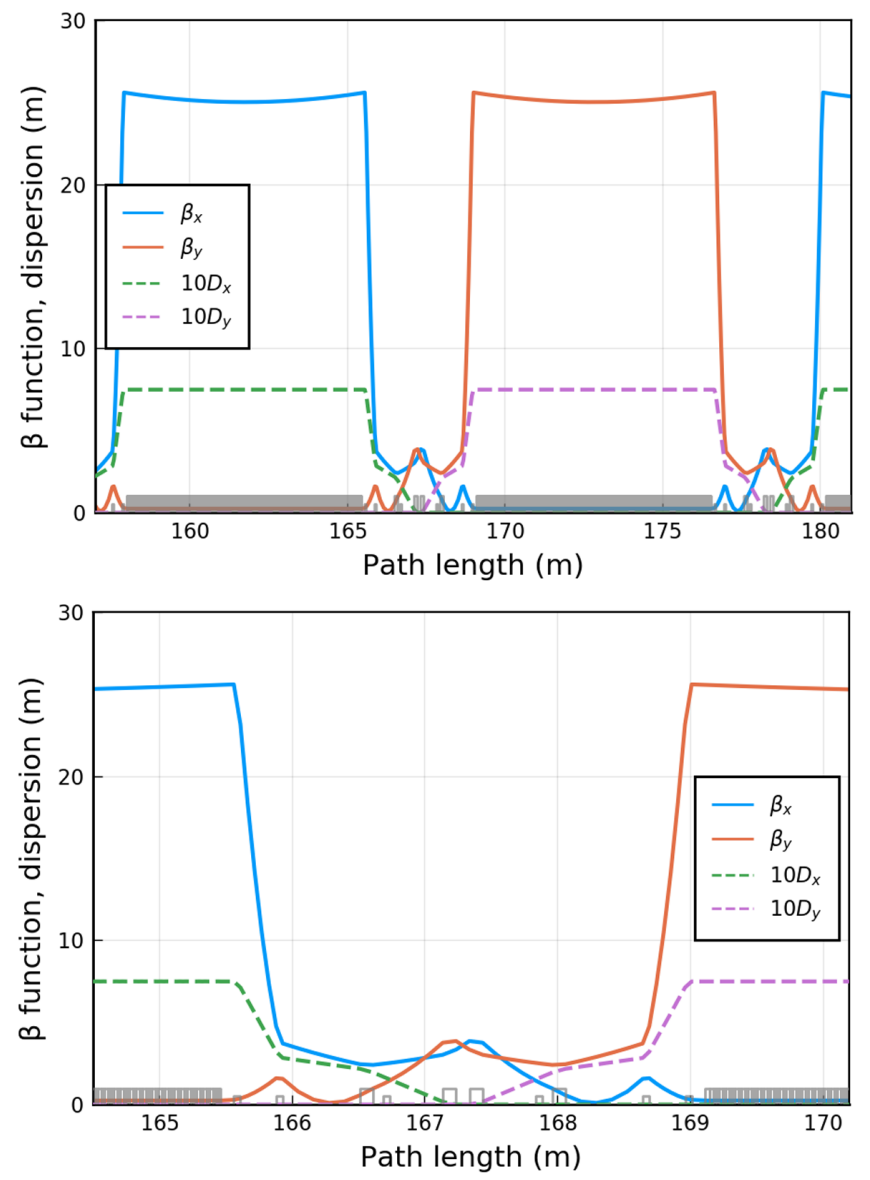

FIG. 4. Twiss functions in the wiggler and in the transition between wigglers.

not have to be small as long as $D^{\prime}$ is kept small. In addition, to avoid the large closed orbit distortion by large dispersion, we finally set the dispersion in the wiggler section to $75 \mathrm{~cm}$, which works well for both chromaticity correction and dynamic aperture.

TABLE II. Parameters of the ring cooler.

Circumference $[\mathrm{m}]$

Length of cooling section [m]

Average $\beta$ function in cooling section [m]

Dispersion in arc $[\mathrm{cm}]$

Wiggler field [T]

Length of wiggler [m]

Bend radius of wiggler [m]

Poles in wiggler

Wiggler period $[\mathrm{cm}]$

$\beta$ function in wiggler [m]

Maximum dispersion in wiggler [cm]

Number of wiggler magnets

Tune $\left(Q_{x} / Q_{y}\right)$

Chromaticity before correction (x/y)

Momentum compaction factor $\alpha$

Natural emittance (x/y) [nm]

Natural momentum spread

\begin{tabular}{c}
449.079 \\
170 \\
$170 / 280$ \\
18.5 \\
1.88 \\
7.44 \\
0.246 \\
158 \\
4.8 \\
25 \\
75 \\
16 \\
$59.92 / 59.85$ \\
$117.8 /-114.4$ \\
$-3.21 \times 10^{-3}$ \\
$3.1 / 3.1$ \\
$2.6 \times 10^{-4}$ \\
\hline
\end{tabular}


In order to minimize the nonlinear effect from the sextupole, the phase advance over a wiggler pair needs to be $\pi / 2$ or $3 \pi / 2$ so that the focusing kicks caused by the sextupole are compensated in the next pair and orbit kicks are compensated in the second next pair. This phase advance is dominated by the beta function in the nonwiggle plane, which is set to be the bend radius and therefore keeps constant. The wiggler field strength needs to be optimized to fulfill the above condition. Since wiggler field also affects the damping and IBS, we finally set the magnetic field of the wiggler to $1.88 \mathrm{~T}$, which, based on the simulations, results in good beam emittance and momentum spread. The preliminary parameters of the ring cooler are summarized in Table II.

\section{BEAM PARAMETERS OF THE COOLER}

\section{A. Emittance and momentum spread}

In the ring cooler, the cooling performance is directly determined by achieved electron beam quality. Based on the lattice design, the equilibrium electron beam parameters can be calculated by considering the radiation damping, quantum excitation and the IBS effects. In addition, since the electron beam will be stored in the ring and continuously interact with hadrons, the heating effect on electrons by the hadron beam also needs to be considered, which is referred to here as the beam-beam scattering (BBS) effect. The differential equation of the emittance of electron beam is given by

$$
\frac{d \epsilon}{d t}=\left(-2 \lambda_{\mathrm{damp}}+\lambda_{\mathrm{IBS}}+\lambda_{\mathrm{BBS}}\right) \epsilon+C_{q},
$$

where $\lambda_{\text {damp }}$ is the radiation damping rate, $C_{q}$ is the factor of quantum excitation, $\lambda_{\mathrm{IBS}}$ and $\lambda_{\mathrm{BBS}}$ are the heating rates from IBS and BBS, respectively. The equation of momentum spread has the same form as Eq. (1). It is known that the radiation damping rate $\lambda_{\text {damp }}$ and the factor of quantum excitation $C_{q}$ only depend on the ring lattice, while the heating rates from IBS $\lambda_{\mathrm{IBS}}$ and BBS $\lambda_{\mathrm{BBS}}$ depend on beam parameters dynamically. In order to get the equilibrium beam parameters in the ring cooler, a simulation code was developed which allows to perform turn-by-turn tracking.

In the simulation, the radiation damping rates are calculated from the radiation integrals [16] based on the optics of the ring. The factor of quantum excitation can be obtained by $C_{q}=2 \lambda_{\text {damp }} \epsilon_{\text {nat }}$, where $\epsilon_{\text {nat }}$ is the natural emittance of electron beam. The Bjorken-Mtingwa IBS model [17] with horizontal and vertical dispersion is used in the code. The code also uses fast algorithm for IBS calculation in the absence of $x-y$ coupling $[18,19]$. In our case, the IBS heating rates for electrons are close to the radiation damping rates due to the small beam emittance caused by the strong damping effect. As a result, the IBS effect is important for accurate calculation of electron beam dynamics in the simulation.
Similar to the cooling effect, the BBS effect is caused by the Coulomb interaction between the electrons and hadrons. Starting with the Boltzmann transport equation, the model for such heating of electrons due to collisions with hadrons was developed using the full Landau collision integral [20] that allows for different temperatures in all three dimensions. Considering that both the electron beam and hadron beam have Gaussian velocity distribution with standard deviation $\sigma_{v_{e}}$ and $\sigma_{v_{i}}$, the horizontal heating rate for electrons can be obtained as

$$
\begin{aligned}
\left\langle\dot{v}_{x}^{2}\right\rangle= & \frac{\gamma_{e i} n_{i}}{(2 \pi)^{3} \sigma_{v_{e x}} \sigma_{v_{e y}} \sigma_{v_{e s}} \sigma_{v_{i x}} \sigma_{v_{i y}} \sigma_{v_{i s}}} \\
& \times \frac{L_{\mathrm{cool}}}{C} \int d^{3} u\left\{\frac{u^{2}-u_{x}^{2}}{u^{3}} \frac{1}{\sigma_{v_{e x}}^{2}} I_{x, 2} I_{y, 0} I_{s, 0}\right. \\
& -\frac{u_{x} u_{y}}{u^{3}} \frac{1}{\sigma_{v_{e y}}^{2}} I_{x, 1} I_{y, 1} I_{s, 0} \\
& \left.-\frac{u_{x} u_{s}}{u^{3}} \frac{1}{\sigma_{v_{e s}}^{2}} I_{x, 1} I_{y, 0} I_{s, 1}\right\}
\end{aligned}
$$

where $\gamma_{e i}=\frac{e_{e}^{2} e_{i}^{2} \ln \Lambda}{4 \pi \varepsilon_{0}^{2} m_{e}^{2}}, e_{e}, e_{i}$ are the charges of electron and hadron, $\ln \Lambda$ is the Coulomb logarithm, $n_{i}$ is the density of hadron beam, $L_{\text {cool }}$ is the length of cooling section and $C$ is the circumference of the ring, $u=v_{e}-v_{i}$ is the velocity difference between the electron and the hadron. The simplified integrals $I_{m, n}=I\left(\frac{1}{2 \sigma_{v_{e m}^{2}}}, \frac{1}{2 \sigma_{v_{i m}^{2}}}, u_{m}, n\right) \quad$ are described by

$$
\begin{aligned}
I(\alpha, \beta, u, 0) & =\sqrt{\frac{\pi}{\alpha+\beta}} \exp \left(-\frac{\alpha \beta}{\alpha+\beta} u^{2}\right) \\
I(\alpha, \beta, u, 1) & =-\frac{\beta u}{\alpha+\beta} I(\alpha, \beta, u, 0) \\
I(\alpha, \beta, u, 2) & =\left[\frac{1}{2(\alpha+\beta)}+\frac{\beta^{2} u^{2}}{(\alpha+\beta)^{2}}\right] I(\alpha, \beta, u, 0) .
\end{aligned}
$$

The final BBS heating rate is $\lambda_{\mathrm{BBS}, x}=\left\langle\dot{v}_{x}^{2}\right\rangle / 2 \sigma_{v x}^{2}$. The vertical and longitudinal BBS rates have the form similar to Eq. (2). This model was benchmarked with the IBS effect as well as other simplified models; more details can be found in Ref. [21]. Since the beta functions at the cooling section are large, the evolution of the beam distribution along the cooling section is small and thus not included in the BBS calculation.

The simulation code, which includes all these effects described above, was used for calculation of the electron beam parameters. Based on the ring lattice and proton beam parameters listed in Table I, the evolution of the electron beam in the ring cooler with two sets of arbitrary initial parameters is shown in Fig. 5. After several thousand turns, the electron beam converges to an equilibrium state. The equilibrium beam parameters are listed in Table III. We can see that the longitudinal BBS rate is close to the IBS 


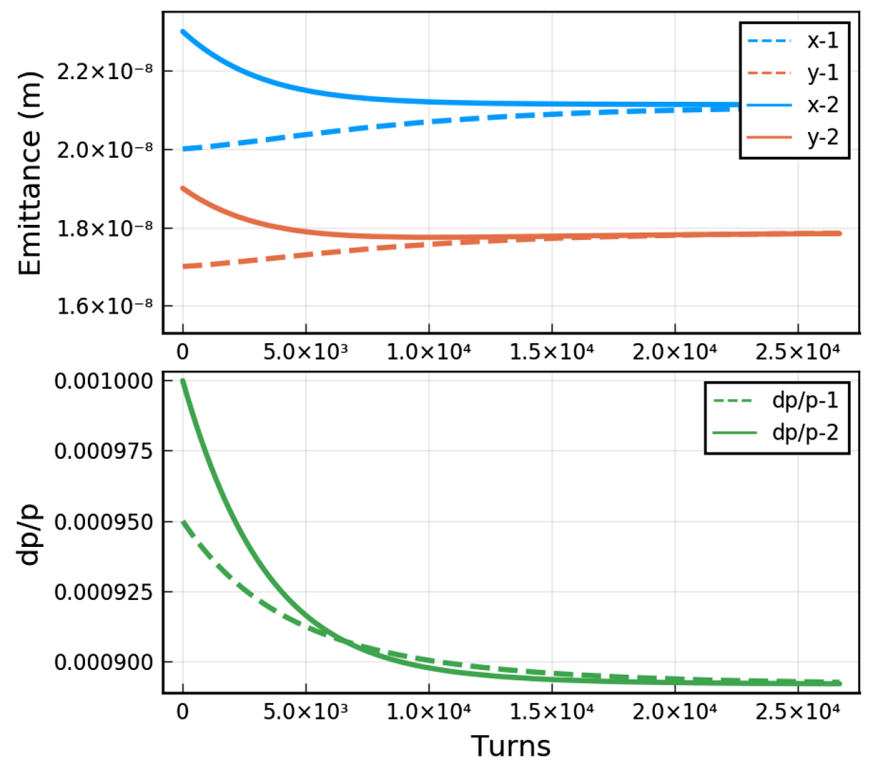

FIG. 5. Evolution of the electron beam parameters in the ring cooler with two sets of arbitrary initial parameters.

heating rate which means the BBS is important for the beam dynamics in the ring cooler.

\section{B. Dynamic aperture}

In the ring cooler, there are two families of sextupoles to minimize the chromaticity. The nonlinear behavior of the ring is minimized by changing the sextupole strength and the phase advance across the mid-arc and mid-wiggler adapters. Meanwhile, the off-energy closed orbit deviation is also minimized. The dynamic aperture is calculated with particle tracking using ELEGANT [22]. In the calculation, the rf cavity

TABLE III. Electron beam parameters in the ring cooler.

\begin{tabular}{lc}
\hline \hline Beam energy [MeV] & 149.8 \\
\hline Relativistic factor $\gamma$ & 293.1 \\
Number of electrons per bunch & $3 \times 10^{11}$ \\
Peak current [A] & 48.3 \\
Number of bunches & 135 \\
Average current [A] & 4.4 \\
rms emittance (x/y) [nm] & $21 / 18$ \\
rms momentum spread & $8.9 \times 10^{-4}$ \\
rms bunch length [cm] & 12 \\
Required rf voltage [kV] & 6.1 \\
Synchrotron tune $Q_{s}$ & $1.63 \times 10^{-3}$ \\
Maximum space charge (x/y) & $0.19 / 0.21$ \\
Damping rates $(\mathrm{x} / \mathrm{y} / \mathrm{s})\left[\mathrm{s}^{-1}\right.$ ] & $32 / 32 / 64$ \\
IBS rates (x/y/s) [s ${ }^{-1}$ ] & $54 / 53 / 68$ \\
BBS rates (x/y/s) [s ${ }^{-1}$ ] & $-0.4 / 1.0 / 49$ \\
Dynamic aperture $(\mathrm{x} / \mathrm{y} / \mathrm{s})$ & $\sigma / 6 \sigma / 13 \sigma$ \\
Momentum aperture & $0.79 \%$ \\
Quantum lifetime [hour] & 2.8 \\
Touschek lifetime [s] & $<55$ \\
\hline \hline
\end{tabular}
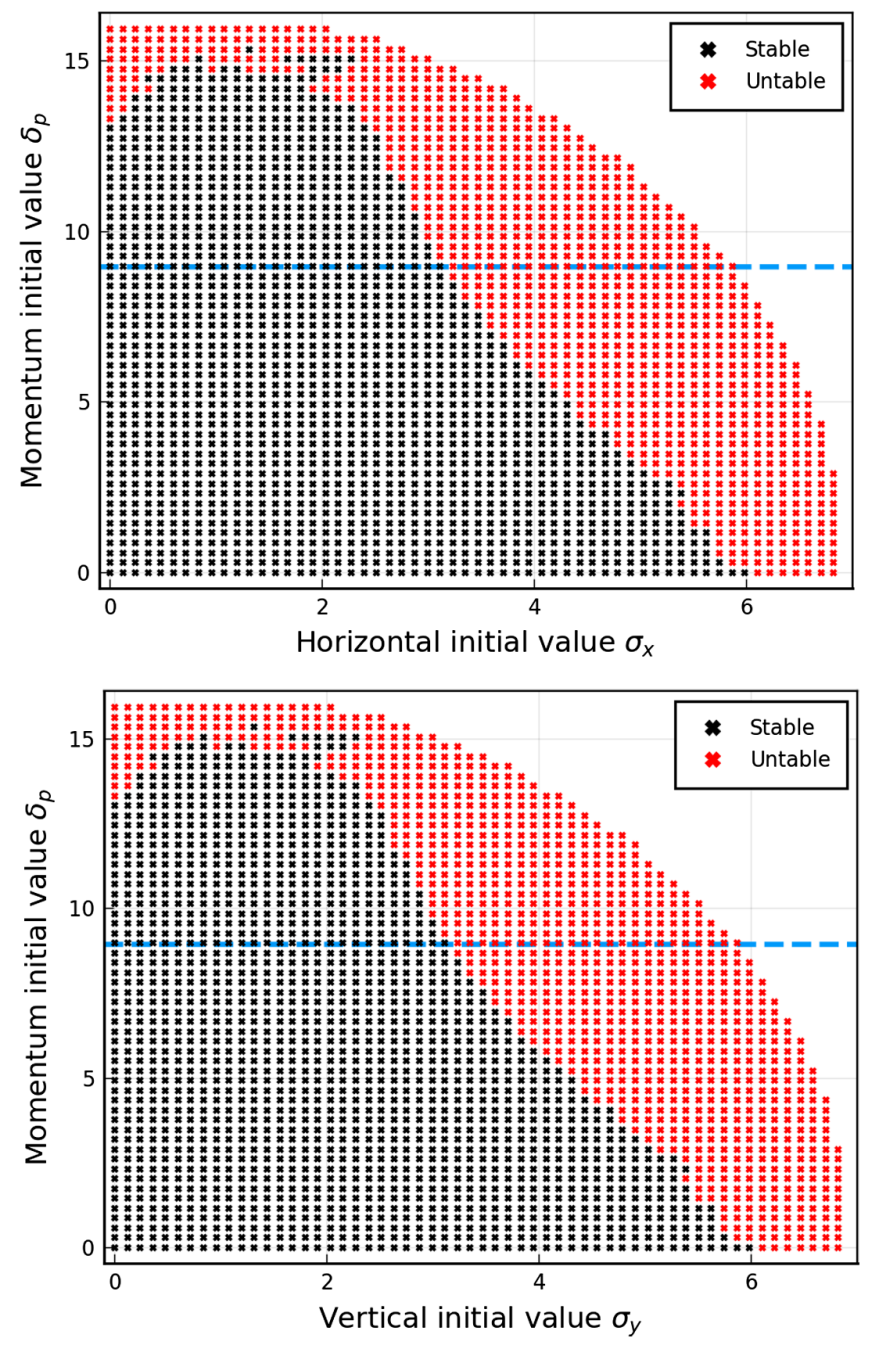

FIG. 6. Dynamic aperture tracking result by ELEGANT (black: survived particles; red: lost particles). The dashed line is the rfmomentum aperture $\left(\epsilon_{x, \mathrm{rms}}=21 \mathrm{~nm}, \epsilon_{y, \mathrm{rms}}=18 \mathrm{~nm}, \delta_{p, \mathrm{rms}}=\right.$ $\left.8.9 \times 10^{-4}\right)$.

is included to match the longitudinal beam emittance. The coordinates of particles are recorded and tracked in 1000 turns ( $\sim$ one synchrotron period). Then the dynamic aperture is defined by the surviving particles. The tracking result is shown in Fig. 6, in which the survived particles are marked in black and lost in red. The axes are in units of sigmas. We see that the maximum dynamic apertures in horizontal, vertical and momentum planes are about $6 \sigma_{x}, 6 \sigma_{y}$ and $13 \delta_{p}$, respectively. In addition, the rf-momentum aperture is about $0.79 \%$, as shown by the dashed line in Fig. 6 .

\section{Beam lifetime}

For the ring cooler with strong radiation damping, the large angle Coulomb scattering is the main effect for the beam loss in longitudinal plane. Based to the results shown in Fig. 6, the momentum acceptance is close to the rf-momentum aperture, which is $0.79 \%$. Then the Touschek 
lifetime is estimated to be 55 seconds. However, the value of momentum acceptance is quite optimistic since the systematic field errors to magnet are not considered in the dynamic aperture tracking. So the Touschek lifetime should be shorter, but it can still be optimized.

Since the transverse dynamic aperture is not very large, the quantum lifetime of the ring cooler needs to be checked. Without consideration of collective effects, a stationary distribution of the electron beam in a storage ring can be estimated based on the heating effects, quantum fluctuation and the radiation damping. As a result of the physical and dynamic aperture, the distribution is cut off with a steady loss of particle, which defines the quantum lifetime. The 1D quantum lifetime has been analyzed by Piwinski [23]. Here we give the derivation of the 3D quantum lifetime. Based on the same method as Piwinski, the three-dimensional continuity equation becomes

$$
\frac{\partial w}{\partial t}+\frac{\partial I_{x}}{\partial \epsilon_{x}}+\frac{\partial I_{y}}{\partial \epsilon_{y}}+\frac{\partial I_{p}}{\partial \delta_{p}^{2}}=0
$$

where $w=w\left(\epsilon_{x}, \epsilon_{y}, \delta_{p}^{2}, t\right)$ is the beam distribution function and $I$ is the flux of density. The beam lifetime is defined by the decay of beam density:

$$
\begin{aligned}
\frac{1}{\tau} & =-\frac{1}{N} \frac{d N}{d t}=-\frac{1}{d t} \iiint_{V} w d \epsilon_{x} d \epsilon_{y} d \delta_{p}^{2} \\
& =\iiint_{V} \frac{\partial I_{x}}{\partial \epsilon_{x}}+\frac{\partial I_{y}}{\partial \epsilon_{y}}+\frac{\partial I_{p}}{\partial \delta_{p}^{2}} d \epsilon_{x} d \epsilon_{y} d \delta_{p}^{2} \\
& =\frac{1}{\tau_{x}}+\frac{1}{\tau_{y}}+\frac{1}{\tau_{p}},
\end{aligned}
$$

where $V=f\left(\epsilon_{x}, \epsilon_{y}, \delta_{p}^{2}\right)$ is the beam acceptance defined by the $3 \mathrm{D}$ aperture, which we assume has an ellipsoid shape:

$$
\frac{\epsilon_{x}}{A_{x}^{2} \hat{\epsilon}_{x}}+\frac{\epsilon_{y}}{A_{y}^{2} \hat{\epsilon}_{y}}+\frac{\delta_{p}^{2}}{A_{p}^{2} \hat{\delta}_{p}^{2}}=1,
$$

where $A_{x}=a / \hat{\sigma}_{x}, A_{y}=b / \hat{\sigma}_{y}$ and $A_{p}=c / \hat{\delta}_{p}$ are the ratios between aperture and rms beam size in horizontal, vertical and longitudinal planes, respectively. The lifetime, for example $\tau_{x}$, can be written by

$$
\frac{1}{\tau_{x}}=\int_{0}^{A_{y}^{2} \hat{\epsilon}_{y}} \int_{0}^{A_{p}^{2} \hat{\delta}_{p}^{2}\left(1-\frac{\epsilon_{y}}{A_{y}^{2} \hat{\varepsilon}_{y}}\right)} I_{x m}\left(\epsilon_{y}, \delta_{p}^{2}\right) d \delta_{p}^{2} d \epsilon_{y}
$$

Comparing with the Fokker-Planck equation in Ref. [23], the beam flux of density is

$$
I_{x}=2 \lambda_{x} \epsilon_{x} M_{x}+\epsilon_{x} M_{x} \partial w / \partial \epsilon_{x},
$$

where $\lambda$ is the effective damping rate and $M$ is the factor of quantum excitation. Considering Gaussian distribution, the stationary beam distribution function $(\partial w / \partial t=0)$ is

$$
w\left(\epsilon_{x}, \epsilon_{y}, \delta_{p}^{2}\right)=\frac{1}{8 \hat{\epsilon}_{x} \hat{\epsilon}_{y} \hat{\delta}_{p}^{2}} \exp \left(-\frac{\epsilon_{x}}{2 \hat{\epsilon}_{x}}-\frac{\epsilon_{y}}{2 \hat{\epsilon}_{y}}-\frac{\delta_{p}^{2}}{2 \hat{\delta}_{p}^{2}}\right),
$$

where $\hat{\epsilon}_{x}, \hat{\epsilon}_{y}$ and $\hat{\delta}_{p}$ are the rms beam emittance and momentum spread, which can be written into $\hat{\epsilon}_{x, y}=$ $M_{x, y} / 4 \lambda_{x, y}$ and $\hat{\delta}_{p}^{2}=M_{p} / 4 \lambda_{p}$. Approximately, the maximum flux $I_{x m}=\int_{0}^{\epsilon_{x m}} \partial I_{x} / \partial \epsilon_{x} d \epsilon_{x}$ can be derived by

$$
\frac{I_{x m}}{\epsilon_{x m}} \int_{-\infty}^{\epsilon_{x m}} \exp \left(\frac{\epsilon_{x}}{2 \hat{\epsilon}_{x}}\right) d \epsilon_{x}=M_{x} w\left(\epsilon_{x}=0, \epsilon_{y}, \delta_{p}^{2}\right) .
$$

Considering $\epsilon_{x m}=A_{x}^{2} \hat{\epsilon}_{x}\left(1-\frac{\epsilon_{y}}{A_{y}^{2} \hat{\epsilon}_{y}}-\frac{\delta_{p}^{2}}{A_{p}^{2} \hat{\delta}_{p}^{2}}\right)$ and substituting Eqs. (9) and (10) into Eq. (7), $\tau_{x}$ can be calculated. With the help of MATHEMATICA, the final form of the 3D quantum lifetime is

$$
\frac{1}{\tau}=A_{x}^{2} A_{y}^{2} A_{p}^{2}\left(k_{x} e^{\frac{-A_{x}^{2}}{2}}+k_{y} e^{\frac{-A_{y}^{2}}{2}}+k_{p} e^{\frac{-A_{p}^{2}}{2}}\right) .
$$

where

$$
\begin{aligned}
k_{x, y, p}= & \frac{1}{\left(A_{p, x, y}^{2}-A_{x, y, p}^{2}\right)^{2}\left(A_{y, p, x}^{2}-A_{x, y, p}^{2}\right)^{2}} \\
& \times\left\{\lambda_{x, y, p}\left[A_{x, y, p}^{2}\left(A_{x, y, p}^{2}-A_{y, p, x}^{2}-A_{p, x, y}^{2}\right)+A_{y, p, x}^{2} A_{p, x, y}^{2}\right]\right. \\
& +2 A_{x, y, p}^{2}\left(2 \lambda_{x, y, p}-\lambda_{y, p, x}-\lambda_{p, x, y}\right) \\
& +2 A_{y, p, x}^{2}\left(\lambda_{p, x, y}-\lambda_{x, y, p}\right) \\
& \left.+2 A_{p, x, y}^{2}\left(\lambda_{y, p, x}-\lambda_{x, y, p}\right)\right\}
\end{aligned}
$$

The effective damping rate is $\lambda=\lambda_{\text {damp }}-\lambda_{\mathrm{IBS}} / 2-\lambda_{\mathrm{BBS}} / 2$, which depends on the radiation damping and heating effects. If the apertures in the other two dimensions are very large, the formula agrees with the 1D form: $\tau=\exp \left(\frac{A^{2}}{2}\right) /\left(\lambda A^{2}\right)$, which is consistent with Ref. [23]. For the ring cooler, the quantum lifetime is limited by transverse dynamic aperture

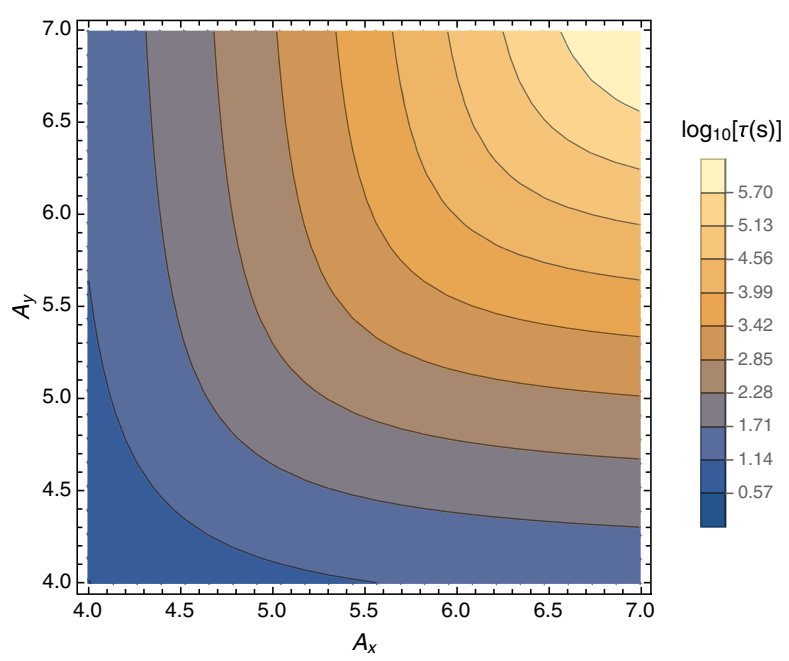

FIG. 7. The dependency of the quantum lifetime on the horizontal and vertical apertures with $A_{p}=8.8$. $\left(\lambda_{x} / \lambda_{y} / \lambda_{p}=\right.$ 5.2/5.0/5.5 s $\left.{ }^{-1}\right)$. 
and momentum acceptance. Figure 7 gives the dependency of the quantum lifetime on the transverse aperture with $A_{p}=8.8$. With the present aperture of the ring cooler $A_{x} / A_{y} / A_{p}=6 / 6 / 8.8$, the quantum beam lifetime is about 2.8 hours.

\section{Impedance and instabilities}

Because of the high beam density the collective instabilities require attention, especially the coherent synchrotron radiation (CSR) impedance caused by such long wigger section. In this paper we use only the resistive wall impedance of the vacuum chamber to estimate instability threshold, in order to compare it with the CSR contribution in the wigglers, which is a unique contribution of presented ring design. More accurate calculations should include other vacuum chamber contributions such as kickers, bellows, BPMs, etc., and will be included during the engineering design stage of such a cooler. In this section, we only discuss the microwave instabilities of the single bunch.

Based on bunch parameters, the threshold impedance for the longitudinal single-bunch instability is given by Boussard criterion [24]

$$
\left|\frac{Z}{n}\right|^{\mathrm{thr}}=\frac{\sqrt{2 \pi} \alpha E \delta_{p}^{2} \sigma_{s}}{e I_{b} R}=49 \mathrm{~m} \Omega,
$$

where $\alpha$ is the momentum compaction factor, $R$ is the average bending radius and $I_{b}$ is the average bunch current. Assuming a circular copper chamber of radius $b=3 \mathrm{~cm}$, the effective longitudinal resistive wall impedance can be obtained:

$$
\left|\frac{Z}{n}\right|_{\mathrm{eff}}^{\mathrm{RW}}=\frac{(1-i) C}{2 \pi b \sigma_{c} \delta_{\mathrm{skin}} n_{\mathrm{eff}}}=(1-i) 20 \mathrm{~m} \Omega,
$$

where $C$ is the ring circumference, $\sigma_{c}$ is the conductivity of the pipe wall, $\delta_{\text {skin }}$ is the skin depth and $n_{\text {eff }} \sim C /\left(2 \pi \sigma_{s}\right)=596$ is the effective harmonic. The effective wall impedance is below the threshold impedance. Besides resistive wall impedance, the coherent synchrotron radiation (CSR) effect is also important for the longitudinal instability in a electron storage ring. Usually, the CSR effect can be significantly suppressed by a shielding provided by two paralleled conducting plates. Since we have a long wiggler section, the CSR effect needs to be studied seriously. Applying the model of the wiggler with finite length from Ref. [25], the CSR impedance from wigglers can be calculated numerically. Based on the parameters list in Table II, the CSR impedance for a single wiggler with a shielding gap of 2 and $4 \mathrm{~cm}$ are calculated, as shown in Fig. 8. The effective CSR impedance at the resonance frequencies for both cases is about $|Z / n|_{\mathrm{eff}}^{\mathrm{CSR}}=0.1 \Omega$. In the ring cooler with a long wiggler section, the CSR impedance is much larger than the threshold. For the electron bunch, the highest frequencies might become unstable. Although we have not had a chance to do the simulations to investigate the microwave instabilities caused by CSR, with

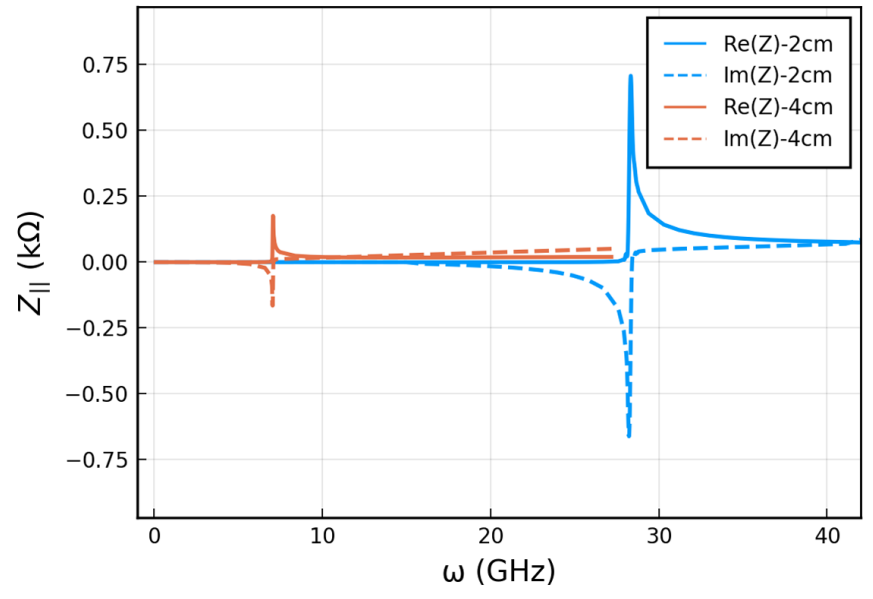

FIG. 8. CSR impedance of a single wiggler with the shielding gap of 2 and $4 \mathrm{~cm}$.

the resonance frequency corresponding to the shielding gap, one can spread the peak value out by smoothly varying the gap in the wiggler, thus reducing the impedance. A detailed study of the instabilities is left for future work.

The threshold for the transverse mode-coupling instability is

$$
Z_{\perp}^{\mathrm{thr}}=\frac{2 E w_{0} Q_{s} \tau_{L}}{e I_{b} \bar{\beta}_{\perp}}=2.0 \mathrm{k} \Omega / \mathrm{m}
$$

where $Q_{s}$ is the synchrotron tune, $\tau_{L}$ is the full bunch length and $\bar{\beta}_{\perp}$ is the average of the $\beta$ function. The transverse resistive wall impedance can be calculated from the Panofsky-Wenzel theorem

$$
Z_{\perp}^{\mathrm{RW}}=\frac{2 R}{b^{2}}\left|\frac{Z}{n}\right|_{\mathrm{eff}}^{\mathrm{RW}}=(1-i) 3.2 \mathrm{k} \Omega / \mathrm{m} .
$$

It shows the transverse resistive wall impedance is slightly above the threshold. This problem can be solved by choosing a larger beam pipe radius. However, we only give an estimate of the resistive wall and CSR impedance in the ring cooler, more accurate calculations need to include other elements. Moreover, details about the beam instabilities and dynamics on single bunch and multibunches need to be further studied through simulation.

In summary, the final electron beam parameters are summarized in Table III. The bunch charge of electron beam is $48 \mathrm{nC}$ and the total charge in the ring is $6.5 \mu \mathrm{C}$. At such high beam intensity the peak space charge tune shift is about 0.2. These numbers are challenging and should be further optimized and improved during the design stage of such ring-based electron cooler. But so far, all parameters look feasible.

\section{COOLING SIMULATION}

In order to estimate the cooling performance of the ring cooler, a simulation code was developed, in which several 
effects relevant to the cooling approach are included. The Bjorken-Mtingwa IBS model and the 3D nonmagnetized cooling force are included and benchmarked with the experimental results in Ref. [26]. In addition, the dispersion effects on electron and ion beams are also included, which are used to redistribute the cooling rates between the horizontal and the longitudinal direction [27-29].

At high energy, the horizontal angular spread of electron beam in the rest frame is much larger than in the longitudinal plane, which creates unbalanced cooling rates between the horizontal and longitudinal cooling. Unlike conventional low-energy electron coolers, we do not use the magnetized cooling method in our design to enhance the transverse cooling. Instead, we introduce the dispersions both for the hadron and electron beams to increase the horizontal cooling rate at the expense of the longitudinal cooling. As we know, dispersion on ions couples the horizontal coordinate with the longitudinal momentum, thus the cooling effect on momentum spread can be transferred to the horizontal plane. Even though the change of beam density caused by dispersion will reduce the cooling rates in all three dimensions, the horizontal cooling rate still can be enhanced by the coupling effect, as long as the dispersion is not too large.

Besides the dispersion on ion beam, we can also apply dispersion on the electron beam in a ring-based cooler, which is also helpful to the cooling redistribution effect. In the simulation code, we include the electron dispersion and the dispersive cooling force is calculated based on the new electron beam distribution. We know that the cooling force depends on the electron beam distribution in $6 \mathrm{D}$ phase space:

$$
\boldsymbol{F}=-4 \pi n_{e} m_{e} r_{e}^{2} Z^{2} c^{4} \int L_{c} \frac{\boldsymbol{u}_{\boldsymbol{i}}-\boldsymbol{u}_{\boldsymbol{e}}}{\left|\boldsymbol{u}_{\boldsymbol{i}}-\boldsymbol{u}_{\boldsymbol{e}}\right|^{3}} f_{e}\left(\boldsymbol{u}_{\boldsymbol{e}}\right) d \boldsymbol{u}_{\boldsymbol{e}} .
$$

With the transverse dispersion $D$, position offset $x_{\text {off }}$ and energy offset $\delta_{\text {off }}$, the new density distribution (Gaussian) of the electron beam becomes

$n_{e}(r)=n_{e 0} \exp \left[-\frac{\left(x-x_{\text {off }}-D \delta_{\text {off }}\right)^{2}}{2\left(\epsilon_{x} \beta_{x}+D^{2} \delta_{p}^{2}\right)}-\frac{y^{2}}{2 \epsilon_{y} \beta_{y}}-\frac{s_{0}^{2}}{2 \sigma_{s}^{2}}\right]$,

and the standard form of velocity distribution in the comoving frame is

$$
\begin{aligned}
f_{e}\left(u_{x}, u_{y}, u_{z}\right)= & \frac{1}{(2 \pi)^{3 / 2} \sigma_{1} \sigma_{2} \sigma_{3} \sqrt{1-\rho^{2}}} \exp \left\{-\frac{1}{2\left(1-\rho^{2}\right)}\right. \\
& \times\left[\frac{\left(u_{x}-\bar{u}_{x}\right)^{2}}{\sigma_{1}^{2}}+\frac{\left(u_{z}-\bar{u}_{z}\right)^{2}}{\sigma_{3}^{2}}\right. \\
& \left.\left.-2 \rho \frac{\left(u_{x}-\bar{u}_{x}\right)\left(u_{z}-\bar{u}_{z}\right)}{\sigma_{1} \sigma_{3}}\right]-\frac{\left(u_{y}-\bar{u}_{y}\right)^{2}}{2 \sigma_{2}^{2}}\right\} .
\end{aligned}
$$

The various parameters are given by

$$
\begin{aligned}
\bar{u}_{x} & =-\frac{\gamma \alpha_{x} \epsilon_{x}\left(x-x_{\mathrm{off}}-D \delta_{\mathrm{off}}\right)}{\epsilon_{x} \beta_{x}+D^{2} \delta_{p}^{2}} \\
\bar{u}_{y} & =-\frac{\gamma \alpha_{y} y}{\beta_{y}} \\
\bar{u}_{z} & =\frac{D \delta_{p}^{2}\left(x-x_{\mathrm{off}}\right)+\epsilon_{x} \beta_{x} \delta_{\mathrm{off}}}{\epsilon_{x} \beta_{x}+D^{2} \delta_{p}^{2}} \\
\sigma_{1}^{2} & =\frac{\epsilon_{x} \gamma^{2}}{\beta_{x}}\left(1+\frac{\alpha_{x}^{2} D^{2} \delta_{p}^{2}}{\epsilon_{x} \beta_{x}+D^{2} \delta_{p}^{2}}\right) \\
\sigma_{2}^{2} & =\frac{\epsilon_{y} \gamma^{2}}{\beta_{y}} \\
\sigma_{3}^{2} & =\frac{\delta_{p}^{2} \epsilon_{x} \beta_{x}}{\epsilon_{x} \beta_{x}+D^{2} \delta_{p}^{2}} \\
\rho & =\frac{\alpha_{x} D \delta_{p}}{\sqrt{\epsilon_{x} \beta_{x}+D^{2} \delta_{p}^{2}\left(1+\alpha_{x}^{2}\right)}}
\end{aligned}
$$

where $\alpha_{x, y}$ and $\beta_{x, y}$ are the Twiss parameters. We see that the effective momentum spread of electron beam $\sigma_{3}$ is reduced by the dispersion, which results in stronger longitudinal cooling. If there is also ion dispersion, the contribution of the longitudinal cooling to the redistribution effect will be greater due to the coupling effect in the ion beam. Based on the beam parameters in Tables I and III, the dependence of the cooling rate on dispersions is studied by simulation, as shown in Fig. 9. In the simulation, only electron cooling is considered and the cooling rates are calculated based on the tracking result of the cooling process. The result shows that the horizontal cooling rate can be increased by electron and ion dispersions, at the expense of the longitudinal cooling. The reduction of vertical cooling rate is due to the change of beam density caused by dispersion. More details about the redistribution of the cooling rates can be found in Refs. [28,29].

In the cooling simulation code, the variation of the electron and proton beam size along the long drift cooling section is included. Actually the space charge effect will also distort the beam distribution, especially for the electron beam. Taking into account the space charge force from electron and proton beams, the evolution of the rms electron beam size and angular spread along cooling section was calculated, as shown in Fig. 10. Since the charge density and bunch length of the electron beam are greater than the proton beam, the distortion is mainly due to the electron beam space charge force. Figure 10 shows that the effect of space charge on the beam distribution is less than $2 \%$, which does not have a significant effect on the cooling force. Therefore, for simplicity, the space charge effect is not included in the present simulation.

Finally, the cooling simulation is performed with the electron beam parameters listed in Table III. Figure 11 

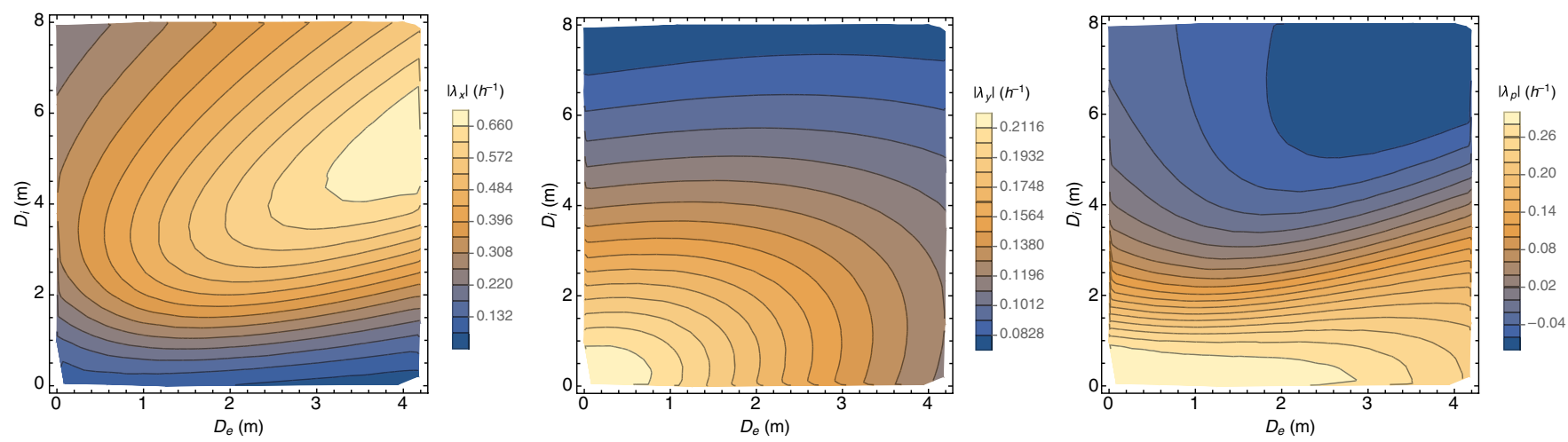

FIG. 9. Dependence of the cooling rate on dispersions.

shows the evolution of the hadron beam transverse and longitudinal emittance with cooling. The beta function of the hadron beam in the center of cooling section is $\beta^{*}=100 \mathrm{~m}$. When there is no dispersion, it shows that there is almost no transverse cooling but a strong longitudinal cooling, which is due to the large difference in cooling gradients. By applying the dispersion both for the proton and electron beam in the cooling section with $D_{i}=$ $2.5 \mathrm{~m}$ and $D_{e}=2 \mathrm{~m}$, the proton beam can be cooled more effectively in the horizontal plane. With cooling, the proton beam emittance is essentially unchanged within two hours, which is close to the requirement in the EIC.

However, dispersion will affect the IBS rate as well as BBS rate on the electron beam, which means that the equilibrium state of the electron beam in the ring cooler
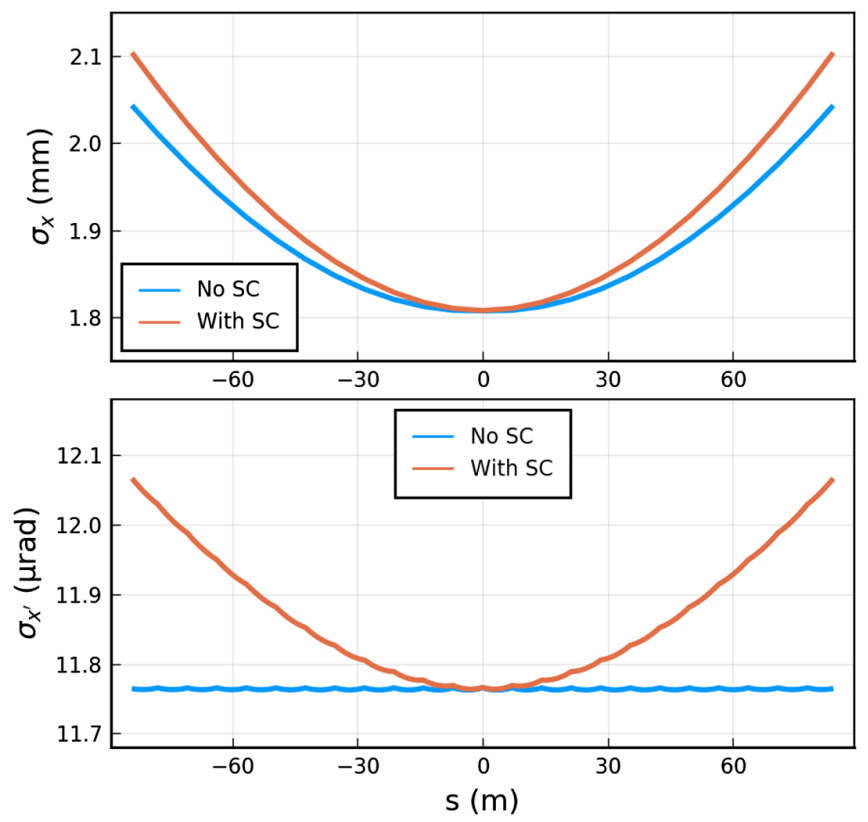

FIG. 10. Evolution of the rms electron beam size and angle spread along the cooling section with and without the space charge effect $\left(\beta_{i}^{*}=100 \mathrm{~m}\right)$. might be different. We did not include this effect so far. Moreover, for the present design, the beam sizes of the proton beam and the electron beam in the cooling section are not well matched. More optimization and matching work need to be done in the future.
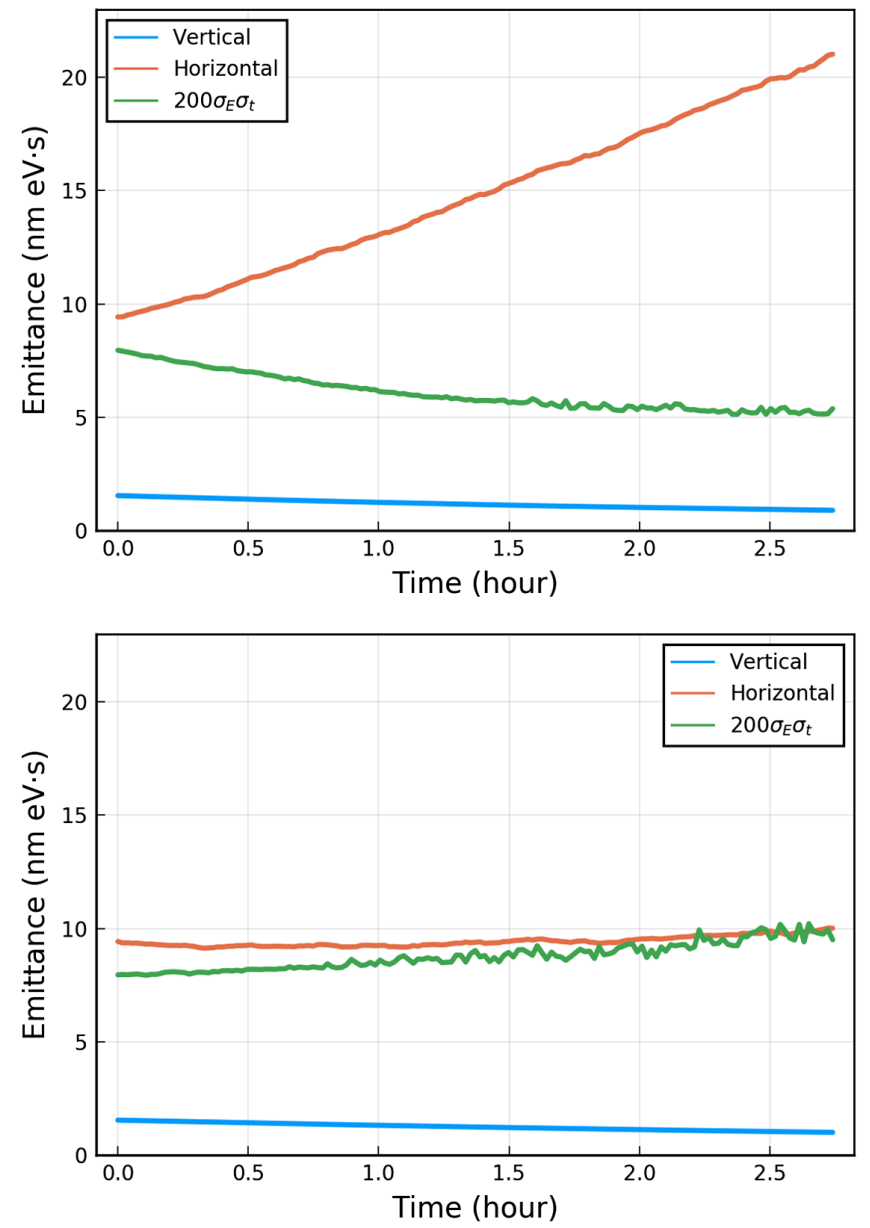

FIG. 11. The evolution of the hadron beam emittance during cooling (upper plot: $D_{i}=0 m, D_{e}=0 \mathrm{~m}$; bottom plot: $\left.D_{i}=2.5 \mathrm{~m}, D_{e}=2 \mathrm{~m}\right)$. 


\section{SUMMARY}

The hadron beam cooling at high energy is an important part of the EIC. In this paper, we present a possible design of such high-energy ring-based electron cooler using bunched electron beam. The electron beam can continuously cool the hadrons while electrons are being cooled by the radiation damping in the storage ring. This approach strongly depends on the design of the electron ring which is described here in detail. The electron beam parameters in such a ring cooler are calculated taking into account several effects, including radiation damping, quantum excitation, IBS and BBS effect. In this paper, we also introduce a generalized treatment of the BBS effect and the 3D quantum lifetime, and make a simple estimation of the beam instabilities in the ring.

Based on such ring cooler design, the cooling performance on the hadron beam is simulated, in which the dispersions of the ion and electron beams in the cooling section are effectively employed to redistribute the cooling rate between the longitudinal and horizontal planes. Although there are still some challenges which have to be addressed during the design stage of such a cooler, it appears that the ring-based electron cooler concept, presented in this paper, offers a viable path for cooling of protons at the top energy in the EIC.

\section{ACKNOWLEDGMENTS}

The authors would like to thank Steve Benson (JLab), Yun Luo and Yongjun Li for useful discussions and suggestions. This work is supported by the U.S. Department of Energy.

[1] G. I. Budker, An effective method of damping particle oscillations in proton and antiproton storage rings, Sov. J. At. En. 22, 438 (1967).

[2] H. Poth, Applications of electron cooling in atomic, nuclear and high-energy physics, Nature (London) 345, 399 (1990).

[3] P. Kienle, The heavy ion cooler and synchrotron ring at GSI, Technical Report, Gesellschaft fuer Schwerionenforschung mbH, 1985.

[4] D. Prasuhn, J. Dietrich, R. Maier, R. Stassen, H. Stein, and H. Stockhorst, Electron and stochastic cooling at COSY, Nucl. Instrum. Methods Phys. Res., Sect. A 441, 167 (2000).

[5] M. Chanel, Leir: The low energy ion ring at CERN, Nucl. Instrum. Methods Phys. Res., Sect. A 532, 137 (2004).

[6] S. Nagaitsev, D. Broemmelsiek, A. Burov, K. Carlson, C. Gattuso, M. Hu, T. Kroc, L. Prost, S. Pruss, M. Sutherland et al., Experimental Demonstration of Relativistic Electron Cooling, Phys. Rev. Lett. 96, 044801 (2006).

[7] A. Fedotov, Z. Altinbas, S. Belomestnykh, I. Ben-Zvi, M. Blaskiewicz, M. Brennan, D. Bruno, C. Brutus, M. Costanzo, A. Drees et al., Experimental Demonstration of Hadron Beam Cooling using Radio-Frequency Accelerated Electron Bunches, Phys. Rev. Lett. 124, 084801 (2020).

[8] F. Willeke, eRHIC pre-conceptual design report, Technical Report, Brookhaven National Laboratory, Upton, NY, 2018.

[9] V. N. Litvinenko and Y. S. Derbenev, Coherent Electron Cooling, Phys. Rev. Lett. 102, 114801 (2009).

[10] D. Ratner, Microbunched Electron Cooling for HighEnergy Hadron Beams, Phys. Rev. Lett. 111, 084802 (2013).

[11] F. Lin, Y. Derbenev, D. Douglas, J. Guo, R. P. Johnson, G. Krafft, V. Morozov, and Y. Zhang, Storage-ring electron cooler for relativistic ion beams, arXiv:1605.02594.

[12] V. Lebedev, S. Nagaitsev, A. Burov, V. Yakovlev, I. Gonin, I. Terechkine, A. Saini, and N. Solyak, Ring-based electron cooling system for the EIC, arXiv:2010.00689.

[13] D. Cline et al., High energy electron cooling to improve the luminosity and lifetime in colliding beam machines, in Proceedings of the Particle Accelerator Conference 1979 [IEEE Trans. Nucl. Sci. 26, 3 (1979)].

[14] M. Gentner, R. Brinkmann, Y. Derbenev, D. Husmann, and C. Steier, On the possibilities of electron cooling for HERA, Nucl. Instrum. Methods Phys. Res., Sect. A 424, 277 (1999).

[15] H. Grote and F. Schmidt, MAD-X-An upgrade from MAD-8, in Proceedings of the 2003 Particle Accelerator Conference, Portland, OR (IEEE, New York, 2003), Vol. 5, pp. 3497-3499.

[16] S. Y. Lee, Accelerator Physics, 3rd ed. (World Scientific, Singapore, 2011), https://www.worldscientific.com/doi/ pdf/10.1142/8335.

[17] J. D. Bjorken and S. K. Mtingwa, Intrabeam scattering, Part. Accel. 13, 115 (1982), https://cds.cern.ch/record/ 140304/files/p115.pdf.

[18] S. Nagaitsev, Intrabeam scattering formulas for fast numerical evaluation, Phys. Rev. STAccel. Beams 8, 064403 (2005).

[19] A. W. Chao, K. H. Mess, M. Tigner, and F. Zimmermann, Handbook of Accelerator Physics and Engineering, 2nd ed. (World Scientific, Singapore, 2013), https://www .worldscientific.com/doi/pdf/10.1142/8543.

[20] R. Hazeltine, Coulomb collision operator, IFS Technical Report No. 1140, 2006.

[21] H. Zhao and M. Blaskiewicz, Electron heating by ions in cooling rings, in Proceedings of the North American Particle Accelerator Conference (NAPAC'19), Lansing, MI, 2019 (JACoW Publishing, Geneva, Switzerland, 2019), https:// doi.org/10.18429/JACoW-NAPAC2019-TUPLM24.

[22] M. Borland, ELEGANT: A flexible SDDS-compliant code for accelerator simulation, Technical Report, Argonne National Laboratory, 2000.

[23] A. Piwinski, Beam losses and lifetime (1985), https://cds .cern.ch/record/165148/files/p432.pdf.

[24] H. Wiedemann, Particle Accelerator Physics (Springer Nature, 2015), https://www.springer.com/gp/book/ 9783319183169.

[25] G. Stupakov and D. Zhou, Analytical theory of coherent synchrotron radiation wakefield of short bunches shielded by conducting parallel plates, Phys. Rev. Accel. Beams 19, 044402 (2016). 
[26] H. Zhao, M. Blaskiewicz, A. V. Fedotov, W. Fischer, X. Gu, D. Kayran, J. Kewisch, C. Liu, S. Seletskiy, V. Schoefer, and P. Thieberger, Cooling simulation and experimental benchmarking for an rf-based electron cooler, Phys. Rev. Accel. Beams 23, 074201 (2020).

[27] Y.S. Derbenev, Theory of electron cooling, arXiv: 1703.09735.
[28] M. Blaskiewicz, Dispersion and electron cooling, Technical Report No. BNL-210932-2019-TECH, Brookhaven National Laboratory, Upton, NY, 2019.

[29] H. Zhao and M. Blaskiewicz, Dispersion effect on the cooling rates, Technical Report No. BNL-220549-2020TECH, Brookhaven National Laboratory, Upton, NY, 2020. 